\title{
A Class of Cayley Digraph Structures Induced by Groups
}

\author{
Anil Kumar V. (Corresponding author) \\ Department of Mathematics, University of Calicut Malappuram \\ Kerala 673 635, India \\ E-mail: anilashwin2003@yahoo.com \\ Parameswaran Ashok Nair \\ Mannaniya College of Arts \& Science Pangode \\ Trivandrum, Kerala 695 609, India \\ E-mail: pashoknair@gmail.com
}

Received: January 4, 2012 Accepted: January 19, 2012 Published: April 1, 2012

doi:10.5539/jmr.v4n2p28 URL: http://dx.doi.org/10.5539/jmr.v4n2p28

\begin{abstract}
In this paper we introduce the Cayley digraph structure. This can be considered as a generalization of Cayley digraph. We prove that all Cayley digraph structures are vertex transitive. Many graph theoretic properties are studied in terms of algebraic properties.
\end{abstract}

Keywords: Cayley digraph, Vertex-transitive graph, Hasse-diagram

AMS Subject Classification Number (2000): 05C25

\section{Digraph Structure}

A binary relation on a set $V$ is a subset $E$ of $V \times V$. A digraph is a pair $(V, E)$ where $V$ is a nonempty set (called vertex set) and $E$ is a binary relation on $V$.

We extend the above definition as follows:

Definition 1.1 Let $V$ be a nonempty set and let $E_{1}, E_{2}, \ldots, E_{n}$ be mutually disjoint binary relations on $G$. Then the $(n+1)$ tuple $G=\left(V ; E_{1}, E_{2}, \ldots, E_{n}\right)$ is called a digraph structure. The elements of $V$ are called vertices and the elements of $\cup E_{i}$ are called edges. In case, a digraph structure with only one binary relation is a digraph. So a digraph structure is a generalization of a digraph.

A digraph structure $\left(V ; E_{1}, E_{2}, \ldots, E_{n}\right)$ is called (i) trivial if $E_{i}=\emptyset$ for all $i$, (ii) reflexive if for all $x \in G,(x, x) \in E_{i}$ for some $i$, (iii) symmetric if $E_{i}=E_{i}^{-1}$ for all $i$, (iv) transitive if for every $i$ and $j, E_{i} \circ E_{j} \subseteq E_{k}$ for some $k$, (v) a hasse diagram if for every positive integer $n \geq 2$ and every $v_{0}, v_{1}, \ldots, v_{n}$ of $V,\left(v_{i}, v_{i+1}\right) \in \cup E_{i}$ for all $i=0,1,2, \ldots, n-1$, implies $\left(v_{0}, v_{n}\right) \notin E_{i}$ for all $i$, and (vi) complete if $\cup E_{i}=V \times V$. A walk of length $k$ in a digraph structure is an alternating sequence $W=v_{0}, e_{0}, v_{1}, \ldots, e_{k-1}, v_{k}$, where $e_{i}=\left(v_{i}, v_{i+1}\right) \in \cup E_{i}$. A walk $W$ is called a path if all the vertices are distinct. We use notation $\left(v_{0}, v_{1}, v_{2}, \ldots, v_{n}\right)$ for the walk $W$. A walk is a called a circuit if its first and last vertices are the same, but no other vertex is repeated. A weak path is a sequence $\left(v_{0}, v_{1}, v_{2}, \ldots, v_{n}\right)$ of distinct vertices of $G$ such that $\left(v_{i}, v_{i+1}\right) \in\left(\cup E_{i}\right) \cup\left(\cup E_{i}\right)^{-1}$. A digraph structure $\left(V ; E_{1}, E_{2}, \ldots, E_{n}\right)$ is called (i) connected (strongly connected) if $v$ is connected to $u$ for all $u, v \in V$, (ii) locally connected iff for every pair of vertices $u, v \in V$ there is a path from $v$ to $u$ whenever there is a path from $u$ to $v$, (iii) semi connected for every pair of vertices $u, v$, there is a path from $u$ to $v$ or a path from $v$ to $u$, and (iv) weakly connected if any two vertices can be joined by a weak path, that is, the digraph structure $\left(V ; E_{1} \cup E_{1}^{-1}, E_{2} \cup E_{2}^{-1}, \ldots, E_{n} \cup E_{n}^{-1}\right)$ is connected. A weakly connected digraph structure $\left(V ; E_{1}, E_{2}, \ldots, E_{n}\right)$ with out any circuits is called a tree.

The distance between two vertices $x$ and $y$ in a digraph structure $G$ is the length of the shortest path between $x$ and $y$, denoted $d(x, y)$. Let $G=\left(V ; E_{1}, E_{2}, \ldots, E_{n}\right)$ be finite connected digraph structure. Then the diameter of $G$ is defined as $d(G)=\max _{u, v \in G} d(x, y)$.

Two digraph structures $\left(V_{1} ; E_{1}, E_{2}, \ldots, E_{n}\right)$ and $\left(V_{2} ; R_{1}, R_{2}, \ldots, R_{n}\right)$ are said to be isomorphic if there exits a bijective function $f: V \rightarrow V$ such that $(x, y) \in \cup E_{i} \Leftrightarrow(f(x), f(y)) \in \cup R_{i}$. An isomorphism of a graph structure onto itself is called an automorphism. A graph structure $\left(V ; E_{1}, E_{2}, \ldots, E_{n}\right)$ is said to be vertex-transitive if, given any two vertices $a$ and $b$ of $V$, there is some graph automorphism $f: V \rightarrow V$ such that $f(a)=b$. Let $\left(V ; E_{1}, E_{2}, \ldots, E_{n}\right)$ be a graph structure and let 
$v \in V$. Then the out-degree of $u$ is $\left|\left\{v \in V:(u, v) \in \cup E_{i}\right\}\right|$ and in-degree of $u$ is $\left|\left\{v \in V:(v, u) \in \cup E_{i}\right\}\right|$.

\section{Cayley Digraph Structure}

Let $G$ be a group and $S$ be a subset of $G$. The cayley digraph of $G$ with respect to $S$ is defined as the digraph $X=(G, E)$, where $E$ is a binary relation on $G$, such that

$$
(x, y) \in E \text { if and only if there is some } s \in S \text {, such that } y=x s \text { (E. Dobson, 2006) }
$$

Informally, the vertices of the cayley digraphs are group elements, and two vertices are connected with an edge if and only if the second vertex is the product of an element from $S$ and the first vertex. The cayley digraph of $G$ with respect to $S$ is denoted by $\operatorname{Cay}(G, S)$. The set $S$ is called the connection set of $\operatorname{Cay}(G, S)$.

We define cayley graph structure as follows:

Definition 2.1 Let $G$ be a group and $S_{1}, S_{2}, \ldots, S_{n}$ be mutually disjoint subsets of $G$. Then cayley digraph structure of $G$ with respect to $S_{1}, S_{2}, \ldots, S_{n}$ is defined as the digraph structure $X=\left(G ; E_{1}, E_{2}, \ldots, E_{n}\right)$, where

$$
E_{i}=\left\{(x, y): x^{-1} y \in S_{i}\right\}
$$

The sets $S_{1}, S_{2}, \ldots, S_{n}$ are called connection sets of $X$. The cayley digraph structure of $G$ with respect to $S_{1}, S_{2}, \ldots, S_{n}$ is denoted by $\operatorname{Cay}\left(G ; S_{1}, S_{2}, \ldots, S_{n}\right)$. In case, a digraph structure with only one connection set is the usual cayley digraph. So a cayley digraph structure is a generalization of the cayley digraph.

\section{Examples of Cayley Digraph Structures}

Example 2.1 Let $G=\mathbb{Z}$, the additive group of integers and let $S_{1}=\{1\}, S_{2}=\{2\}, S_{3}=\{3\}, S_{4}=\{4\}$. Then the cayley digraph structure $\operatorname{Cay}\left(G ; S_{1}, S_{2}, S_{3}, S_{4}\right)$ is shown in figure 1.

Example 2.2 Let $G=\mathbb{Z} \oplus \mathbb{Z}$, the direct sum of the group of integers and let $S_{1}=\{(1,0)\}, S_{2}=\{(0,1)\}, S_{3}=\{(1,1)\}$. Then the cayley digraph structure $\operatorname{Cay}\left(G ; S_{1}, S_{2}, S_{3}\right)$ is shown in figure 2 .

In this paper we may use the following notations. Let Cay $\left(G ; S_{1}, S_{2}, \ldots, S_{n}\right)$ be a cayley digraph structure.

(1) Let $A_{k}$ be the set of all $k$ products of the form $S_{i} S_{j} \cdots S_{k}$. Then [S] is defined as

$$
[S]=\bigcup_{k} A_{k} .
$$

(2) Let $A=\left\{S_{i} \cup S_{i}^{-1}: i=1,2, \ldots, n\right\}$ and let $B_{k}$ be the set of all finite products of elements from $A$ taken $k$ at a time. Then we define

$$
[[S]]=\bigcup_{k} B_{k}
$$

Theorem 2.1 If $G$ is a group and let $S_{1}, S_{2}, \ldots, S_{n}$ are mutually disjoint subsets of $G$, then the cayley digraph structure $\operatorname{Cay}\left(G ; S_{1}, S_{2}, \ldots, S_{n}\right)$ is vertex-transitive.

Proof: Let $a$ and $b$ be any two arbitrary elements in $G$. Define a mapping $\varphi: G \rightarrow G$ by

$$
\varphi(x)=b a^{-1} x \text { for all } x \in G .
$$

This mapping defines a permutation of the vertices of $\operatorname{Cay}\left(G ; S_{1}, S_{2}, \ldots, S_{n}\right)$. It is also a graph automorphism. To see this, note that

$$
\begin{aligned}
(x, y) \in \cup E_{i} & \Leftrightarrow(x, y) \in E_{i} \text { for some } i \\
& \Leftrightarrow x^{-1} y \in S_{i} \text { for some } i \\
& \Leftrightarrow\left(b a^{-1} x\right)^{-1}\left(b a^{-1} y\right) \in S_{i} \text { for some } i \\
& \Leftrightarrow(\varphi(x), \varphi(y)) \in \cup E_{i} .
\end{aligned}
$$

Also we note that $\varphi(a)=b a^{-1} a=b$. Hence $\operatorname{Cay}\left(G ; S_{1}, S_{2}, \ldots, S_{n}\right)$ is vertex-transitive.

Corollary 2.1 Cay $\left(G ; S_{1}, S_{2}, \ldots, S_{n}\right)$ is a trivial graph if and only if $S_{i}=\emptyset$ for all $i$.

Proof: By definition, $\operatorname{Cay}\left(G ; S_{1}, S_{2}, \ldots, S_{n}\right)$ is trivial $\Leftrightarrow E_{i}=\emptyset$ for all $i$. This implies that $S_{i}=\emptyset$ for all $i$.

Corollary 2.2 Cay $\left(G ; S_{1}, S_{2}, \ldots, S_{n}\right)$ is reflexive (each vertex has a loop) if and only if $1 \in \cup S_{i}$. 
Proof: Assume that $\operatorname{Cay}\left(G ; S_{1}, S_{2}, \ldots, S_{n}\right)$ is reflexive. Then for every $x \in G,(x, x) \in E_{i}$ for some $i$. This implies that $x^{-1} x \in S_{i}$ for some $i$. That is, $1 \in S_{i}$ for some $i$.

Conversely, assume that $1 \in S_{i}$ for some $i$. This implies for each $x \in G,(x, x) \in E_{i}$ for some $i$. That is, $(x, x) \in \cup E_{i}$ for all $x \in G$.

Corollary 2.3 Cay $\left(G ; S_{1}, S_{2}, \ldots, S_{n}\right)$ is symmetric if and only if $S_{i}=S_{i}^{-1}$ for all $i$.

Proof:First, assume that $\operatorname{Cay}\left(G ; S_{1}, S_{2}, \ldots, S_{n}\right)$ is symmetric. Let $a \in S_{i}$. Then $(1, a) \in E_{i}$. Since $\operatorname{Cay}\left(G ; S_{1}, S_{2}, \ldots, S_{n}\right)$ is symmetric $(a, 1) \in E_{i}$. This implies that $a^{-1} \in S_{i}$. That is $a \in S_{i}^{-1}$. Hence $S_{i} \subseteq S_{i}^{-1}$. Similarly, we can prove that $S_{i}^{-1} \subseteq S_{i}$

Conversely, if $S_{i}=S_{i}^{-1}$ for all $i$, then we can prove that Cay $\left(G ; S_{1}, S_{2}, \ldots, S_{n}\right)$ is symmetric.

Corollary 2.4 Cay $\left(G ; S_{1}, S_{2}, \ldots, S_{n}\right)$ is a transitive if and only iffor every $i, j, S_{i} S_{j} \subseteq S_{k}$ for some $k$.

Proof: First, assume that $\operatorname{Cay}\left(G ; S_{1}, S_{2}, \ldots, S_{n}\right)$ is transitive. Let $x \in S_{i} S_{j}$ for some $i$ and $j$. Then $x=z_{1} z_{2}$ for some $z_{1} \in S_{i}$ and $z_{2} \in S_{j}$. This implies that $\left(1, z_{1}\right) \in E_{i}$ and $\left(z_{1}, z_{1} z_{2}\right) \in E_{j}$. Since Cay $\left(G, S_{1}, S_{2}, \ldots, S_{n}\right)$ is transitive $\left(1, z_{1} z_{2}\right) \in E_{k}$ for some $k$. That is $z_{1} z_{2} \in S_{k}$. Hence $S_{i} S_{j} \subseteq S_{k}$ for some $k$.

Conversely assume that for each $i, j, S_{i} S_{j} \subseteq S_{k}$ for some $k$. Let $(1, x),(x, y) \in \cup E_{i}$. Then $x \in S_{i}$ for some $i$ and $x^{-1} y \in S_{j}$ for some $j$. This implies that $y \in S_{i} S_{j}$. Since $S_{i} S_{j} \subseteq S_{k}$ for some $k,(1, y) \in S_{k}$.

Corollary $2.5 \operatorname{Cay}\left(G ; S_{1}, S_{2}, \ldots, S_{n}\right)$ is complete if and only if $G=\cup S_{i}$.

Proof:Suppose $\operatorname{Cay}\left(G ; S_{1}, S_{2}, \ldots, S_{n}\right)$ is complete. Then for every $x \in G$, we have $(1, x) \in \cup E_{i}$. This implies that $x \in S_{i}$ for some $i$. This implies that $G=\cup S_{i}$.

Conversely, assume that $G=\cup S_{i}$. Let $x$ and $y$ be two arbitrary elements in $G$ such that $y=x z$. Then $z \in G$. This implies that $z \in S_{i}$ for some $i$. That is, $(1, z) \in \cup E_{i}$. That is $(x, x z)=(x, y) \in \cup E_{i}$. This shows that $\operatorname{Cay}\left(G ; S_{1}, S_{2}, \ldots, S_{n}\right)$ is complete.

Corollary 2.6 Cay $\left(G ; S_{1}, S_{2}, \ldots, S_{n}\right)$ is a union of complete graphs if and only if each $S_{i}$ is a sub group of $G$.

Corollary 2.7 Cay $\left(G ; S_{1}, S_{2}, \ldots, S_{n}\right)$ is connected if and only if $G=[S]$.

Proof: Suppose $\operatorname{Cay}\left(G ; S_{1}, S_{2}, \ldots, S_{n}\right)$ is connected and let $x \in G$. Let

$$
\left(1, y_{1}, y_{2}, \ldots, y_{n}, x\right)
$$

be a path leading from 1 to $x$. Then we have,

$$
y_{1} \in S_{i}, y_{1}^{-1} y_{2} \in S_{j}, \cdots, y_{n}^{-1} x \in S_{k}
$$

This implies that $x \in A$ for some $A \in[S]$. Since $x$ is arbitrary, $G=[S]$.

Conversely, assume that $G=[S]$. Let $x \in G$. Then $x \in S_{i} S_{j} \cdots S_{k}$ for some $i, j, \ldots$ and $k$. This implies that $x=s_{i} s_{j} \ldots s_{k}$ for some $i, j \ldots$ and $k$. Then clearly, $\left(1, s_{i}, s_{i} s_{j}, \ldots, s_{i} s_{j} \ldots s_{k}\right)$ is a path from 1 to $x$. Hence $\operatorname{Cay}\left(G ; S_{1}, S_{2}, \ldots, S_{n}\right)$ is connected.

Corollary 2.8 Cay $\left(G ; S_{1}, S_{2}, \ldots, S_{n}\right)$ is weakly connected if and only if $G=[[S]]$

Suppose $\operatorname{Cay}\left(G ; S_{1}, S_{2}, \ldots, S_{n}\right)$ is weakly connected. Let $x \in G$. Then there exists a weak path say:

$$
\left(1, x_{1}, x_{2}, \ldots, x_{n}, x\right)
$$

from 1 to $x$. This implies that

$$
\begin{aligned}
& x_{1} \in S_{i} \cup S_{i}^{-1} \text { for some } i \\
& x_{1}^{-1} x_{2} \in S_{j} \cup S_{j}^{-1} \text { for some } j \\
& \vdots \\
& x_{n}^{-1} x \in S_{k} \cup S_{k}^{-1} \text { for some } k
\end{aligned}
$$

This implies that $x \in[[S]]$. Since $x$ is arbitrary, $G=[[S]]$.

Conversely, assume that $G=[[S]]$. Let $x$ and $y$ be any two elements in $G$. Then the equation $y=x z$ has a unique solution $z \in G$. This implies that $z \in\left(S_{i} \cup S_{i}^{-1}\right)\left(S_{j} \cup S_{j}^{-1}\right) \cdots\left(S_{k} \cup S_{k}^{-1}\right)$ for some $i, j, \ldots, k$. That is

$$
z=x_{1} x_{2} x_{3} \ldots x_{k}
$$


where $x_{i} \in\left(S_{i} \cup S_{i}^{-1}\right)$. This implies that

$$
\left(1, x_{1}, x_{1} x_{2}, x_{1} x_{2} x_{3}, \ldots, x_{1} x_{2} x_{3} \ldots x_{k}\right)
$$

is a weak path from 1 to $z$. That is

$$
\left(x, x x_{1}, x x_{1} x_{2}, x x_{1} x_{2} x_{3}, \ldots, x x_{1} x_{2} x_{3} \ldots x_{k}\right)
$$

is a weak path from $x$ to $y$. Hence $G$ is weakly connected.

Corollary 2.9 Cay $\left(G ; S_{1}, S_{2}, \ldots, S_{n}\right)$ is a quasi ordered set if and only if

(i) $1 \in S_{1} \cup S_{2} \cdots \cup S_{n}$,

(ii)for every $(i, j), S_{i} S_{j} \subseteq S_{k}$ for some $k$.

Corollary 2.10 Cay $\left(G ; S_{1}, S_{2}, \ldots, S_{n}\right)$ if a partially ordered set if and only if

(i) $1 \in S_{1} \cup S_{2} \cdots \cup S_{n}$,

(ii)for every $(i, j), S_{i} S_{j} \subseteq S_{k}$ for some $k$,

(iii) $\cup\left(S_{i} \cap S_{i}^{-1}\right)=\{1\}$

Corollary 2.11 Let $A_{n}$ is the set of $n$ products of the form $S_{i_{1}} S_{i_{2}} \cdots S_{i_{n}}$. Then Cay $\left(G ; S_{1}, S_{2}, \ldots, S_{n}\right)$ is a hasse- diagram if and only if $C \cap S_{i}=\emptyset$ for all $i$ and for all $C \in A_{n}$.

Proof: Suppose the condition holds. Let $x_{0}, x_{1}, \ldots, x_{n}$ be $(n+1)$ elements in $G$ such that $\left(x_{i}, x_{i+1}\right) \in \cup E_{i}$ for $i=0,1, \ldots, n-$ 1. This implies that

$$
\begin{gathered}
x_{0}^{-1} x_{1} \in S_{i_{1}} \text { for some } i_{1} \\
x_{1}^{-1} x_{2} \in S_{i_{2}} \text { for some } i_{2} \\
\vdots \\
x_{n-1}^{-1} x_{n} \in S_{i_{n}} \text { for some } i_{n}
\end{gathered}
$$

This implies that $x_{0} x_{n}^{-1} \in S_{i_{1}} S_{i_{2}} S_{i_{3}} \cdots S_{i_{n}} \in A_{n}$. Since $C \cap S_{i}=\emptyset$ for all $i$ and for all $C \in A_{n},\left(x_{0}, x_{n}\right) \notin \cup E_{i}$.

Conversely assume that $\operatorname{Cay}\left(G ; S_{1}, S_{2}, \ldots, S_{n}\right)$ is a hasse diagram. We will show that $C \cap S_{i}=\emptyset$ for all $i$ and for all $C \in A_{n}$. Let $S_{i_{1}} S_{i_{2}} S_{i_{3}} \cdots S_{i_{n}}$ be any element in $A_{n}$. Let $x \in S_{i_{1}} S_{i_{2}} S_{i_{3}} \cdots S_{i_{n}}$. Then $x=s_{i_{1}} s_{i_{2}} s_{i_{3}} \ldots s_{i_{n}}$ for some $s_{i_{k}} \in S_{i_{k}}$. This implies that

$$
\left(1, s_{i_{1}}, s_{i_{2}} s_{i_{3}}, \ldots, x\right)
$$

is a path from 1 to $x$. Since $\operatorname{Cay}\left(G ; S_{1}, S_{2}, \ldots, S_{n}\right)$ is a hasse- diagram $x \notin S_{i}$ for any $i$. That is, $A_{n} \cap S_{i}=\emptyset$ for all $i$.

Corollary 2.12 The out-degree of $\operatorname{Cay}\left(G ; S_{1}, S_{2}, \ldots, S_{n}\right)$ is the cardinal number $\left|S_{1} \cup S_{2} \cup \cdots \cup S_{n}\right|$.

Proof: Since $\operatorname{Cay}\left(G ; S_{1}, S_{2}, \ldots, S_{n}\right)$ is vertex-transitive it suffices to consider the out degree of the vertex $1 \in G$. Observe that

$$
\begin{aligned}
\rho(1) & =\{u:(1, u) \in E\} \\
& =\left\{u: u \in S_{i} \text { for some } i\right\} \\
& =S_{1} \cup S_{2} \cup \cdots \cup S_{n}
\end{aligned}
$$

Hence

$$
|\rho(1)|=\left|S_{1} \cup S_{2} \cup \cdots \cup S_{n}\right| .
$$

Corollary 2.13 The in-degree of $\operatorname{Cay}\left(G ; S_{1}, S_{2}, \ldots, S_{n}\right)$ is the cardinal number $\left|S_{1}^{-1} \cup S_{2}^{-1} \cup \cdots \cup S_{n}^{-1}\right|$.

Proof: Since $\operatorname{Cay}\left(G ; S_{1}, S_{2}, \ldots, S_{n}\right)$ is vertex-transitive it suffices to consider the in degree of the vertex $1 \in G$. Observe that

$$
\begin{aligned}
\sigma(1) & =\{u:(u, 1) \in E\} \\
& =\left\{u: u^{-1} \in S_{i} \text { for some } i\right\} \\
& =S_{1}^{-1} \cup S_{2}^{-1} \cup \cdots \cup S_{n}^{-1}
\end{aligned}
$$


Hence

$$
|\sigma(1)|=\left|S_{1}^{-1} \cup S_{2}^{-1} \cup \cdots \cup S_{n}^{-1}\right| .
$$

Corollary 2.14 Cay $\left(G ; S_{1}, S_{2}, \ldots, S_{n}\right)$ is self dual if $G$ is commutative.

Proof: Note that the mapping $\varphi: G \rightarrow G$ defined by $\varphi(x)=x^{-1}$ is a bijective map. Moreover

$$
\begin{aligned}
(x, y) \in \cup E_{i} & \Leftrightarrow(x, y) \in E_{i} \text { for some } i \\
& \Leftrightarrow x^{-1} y \in E_{i} \Leftrightarrow y x^{-1} \in E_{i} \\
& \Leftrightarrow\left(y^{-1}\right)^{-1} x^{-1} \in E_{i} \\
& \Leftrightarrow\left(y^{-1}, x^{-1}\right) \in E \\
& \Leftrightarrow(\varphi(x), \varphi(y)) \in E_{i}^{-1} \subseteq E^{-1} .
\end{aligned}
$$

This implies that $\operatorname{Cay}\left(G ; S_{1}, S_{2}, \ldots, S_{n}\right)$ is isomorphic to its dual.

Corollary 2.15 If $G \backslash\left(S_{1} \cup S_{2} \cup \cdots \cup S_{n}\right)$ is a subgroup of $G$ then $\operatorname{Cay}\left(G ; S_{1}, S_{2}, \ldots, S_{n}\right)$ is a bipartite graph structure.

Corollary 2.16 For $k=1,2,3, \ldots$ let $A_{k}$ be the set of all $k$ products of the form $S_{i_{1}} S_{i_{2}} S_{i_{3}} \cdots S_{i_{k}}$. If Cay $\left(G ; S_{1}, S_{2}, \ldots, S_{n}\right)$ has finite diameter, then the diameter of $\operatorname{Cay}\left(G ; S_{1}, S_{2}, \ldots, S_{n}\right)$ is the least positive integer $n$ such that

$$
G=\bigcup_{A \in A_{n}} A .
$$

Proof: Let $n$ be the smallest positive integer such that $G=\bigcup_{A \in A_{n}} A$. We will show that the diameter of Cay $\left(G ; S_{1}, S_{2}, \ldots, S_{n}\right)$ is $n$. Let $x$ and $y$ be any two arbitrary elements in $G$ such that $y=x z$. Then $z \in G$. This implies that $z \in A$ for some $A$ in $A_{n}$. But then $z$ has a representation of the form $z=s_{i_{1}} s_{i_{2}} \cdots s_{i_{n}}$. This implies that

$$
\left(1, s_{i_{1}}, s_{i_{1}} s_{i_{2}}, \ldots, z\right)
$$

is path of $n$ edges from 1 to $z$. That is

$$
\left(x, x s_{i_{1}}, x s_{i_{1}} s_{i_{2}}, \ldots, y\right)
$$

is a path of length $n$ from $x$ to $y$. This shows that $d(x, y) \leq n$. Since $x$ and $y$ are arbitrary,

$$
\max _{x, y \in G} d(x, y) \leq n
$$

Therefore the diameter of $\operatorname{Cay}\left(G ; S_{1}, S_{2}, \ldots, S_{n}\right)$ is less than or equal to $n$. On the other hand let the diameter of $\operatorname{Cay}\left(G ; S_{1}, S_{2}, \ldots, S_{n}\right)$ be $k$. Let $x \in G$ and $d(1, x)=k$. Then we have $x \in B$ for some $B \in A_{k}$. That is

$$
G=\bigcup_{A \in A_{k}} A .
$$

Now by the minimality of $k$, we have $n \leq k$. Hence $k=n$.

Corollary 2.17 $\operatorname{Cay}\left(G ; S_{1}, S_{2}, \ldots, S_{n}\right)$ is a tree if and only if

(i) $G=[[S]]$

(ii) $1 \notin A$ for all $A \in A_{k}, k=1,2,3, \cdots$

\section{Acknowledgment}

The authors would like to thank the referee for his valuable suggestions which has helped us to improve this paper.

\section{References}

B. Alspach, \& C. Q. Zhang. (1989). Hamilton cycles in cubic Cayley graphs on dihedral groups. Ars Combin., 28, 101-108.

B. Alspach, S. Locke, \& D. Witte. (1990). The Hamilton spaces of Cayley graphs on abelian groups. Discrete Math., 82, 113-126. http://dx.doi.org/10.1016/0012-365X(90)90319-D

B. Alspach, \& Y. Qin. (2001). Hamilton-Connected Cayley graphs on Hamiltonian groups. Europ. J. Combin., 22, 777-787. http://dx.doi.org/10.1006/eujc.2001.0456 
C. Godsil, \& R. Gordon. (2001). Algebraic Graph Theory, Graduate Texts in Mathematics. New York: Springer-Verlag. D. Witte. (1982). On Hamiltonian circuits in Cayley diagrams. Discrete Math., 38, 99-108. http://dx.doi.org/10.1016/0012-365X(82)90174-1

D. Witte. (1986). Cayley digraphs of prime-power order are Hamiltonian. J. Combin. Theory Ser. B, 40, $107-112$. http://dx.doi.org/10.1016/0095-8956(86)90068-7

D. Witte, \& K. Keating. (1985). On Hamilton cycles in Cayley graphs in groups with cyclic commutator subgroup. Ann. Discrete Math., 27, 89-102.

E. Dobson. (2006). Automorphism groups of Metacirculant graphs of order a product of two distinct primes. Combinatorics, Probability and Computing, 15, 105-130. http://dx.doi.org/10.1017/S0963548305007066

G. Sabidussi. (1958). On a class of fxed-point-free graphs. Proc. Amer. Math. Soc., 9, 800-804. http://dx.doi.org/10.1090/S0002-9939-1958-0097068-7

H. B. Richard. (1971). A survey of Binary Systems. New York: Springer-Verlag.

J. B. Fraleigh. (2008). A First course in abstract algebra. Pearson Education.

K. V. Anil. (2011). Double Coset Cayley Digraphs. International Journal of Algebra, 5, (15), 747-753.

K. V. Anil. (2011). A Class of Double Coset Cayley Digraphs induced by Loops. International Journal of Algebra, 5 (22), 1073-1084.

K. V. Anil, \& M. P. Shyama. (2012). A class of double coset cayley digraphs induced by quasigroups. Journal of Mathematics Research, 4 (2) (to appear).

S. B. Akers, \& B. Krishamurthy. (1989). A group theoritic model for symmetric interconnection networks. IEEE Trans. comput., 38, 555-556. http://dx.doi.org/10.1109/12.21148

S. J. Curran, \& J. A. Gallian. (1996). Hamiltonian cycles and paths in Cayley graphs and digraphs-A survey. Discrete Math., 156, 1-18. http://dx.doi.org/10.1016/0012-365X(95)00072-5

S. Lakshmivarahan, J. Jwo, \& S. K. Dhall. (1993). Symmetry in interconnection networks based on Cayley graphs of permutation groups-A survey. Parallel Comput., 19, 361-407. http://dx.doi.org/10.1016/0167-8191(93)90054-O

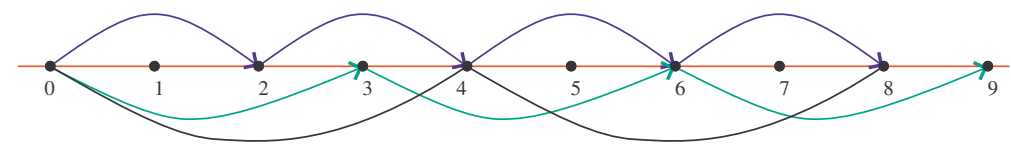

Figure 1. $\operatorname{Cay}\left(G=\mathbb{Z} ; S_{1}=\{1\}, S_{2}=\{2\}, S_{3}=\{3\}, S_{4}=\{4\}\right)$

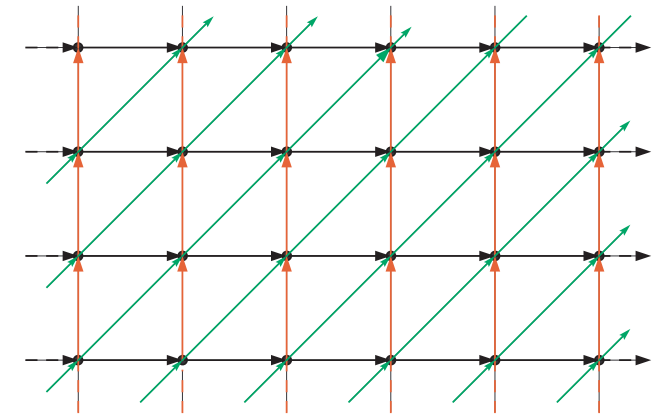

Figure 2. $\operatorname{Cay}\left(G=\mathbb{Z} \oplus \mathbb{Z} ; S_{1}=\{(1,0)\}, S_{2}=\{(0,1)\}, S_{3}=\{(1,1)\}\right)$ 